\title{
Pitting intuitive and analytical thinking against each other: The case of transitivity
}

\author{
Zohar Rusou • Dan Zakay • Marius Usher
}

Published online: 22 February 2013

(C) Psychonomic Society, Inc. 2013

\begin{abstract}
Identifying which thinking mode, intuitive or analytical, yields better decisions has been a major subject of inquiry by decision-making researchers. Yet studies show contradictory results. One possibility is that the ambiguity is due to the variability in experimental conditions across studies. Our hypothesis is that decision quality depends critically on the level of compatibility between the thinking mode employed in the decision and the nature of the decision-making task. In two experiments, we pitted intuition and analytical thinking against each other on tasks that were either mainly intuitive or mainly analytical. Thinking modes, as well as task characteristics, were manipulated in a factorial design, with choice transitivity as the dependent measure. Results showed higher choice consistency (transitivity) when thinking mode and the characteristics of the decision task were compatible.
\end{abstract}

Keywords Choice $\cdot$ Decision making $\cdot$ Judgment and decision making $\cdot$ Dual-process theories

There is considerable agreement among researchers that information in decision making involves two qualitatively different thinking modes: (1) an intuitive mode characterized by fast and parallel processes that are affective, holistic, and associative in nature and (2) a deliberativelanalytical mode characterized by slower processes that are rule based in nature (Epstein, 1994; Evans, 2008; Kahneman, 2003; Sloman, 1996). However, according to Evans, despite the abovementioned agreement, there are different viewpoints regarding

Z. Rusou $(\varangle) \cdot$ D. Zakay $\cdot$ M. Usher

Tel Aviv University, Tel Aviv, Israel

e-mail: zoharrus@gmail.com

Z. Rusou

Open University Israel, Ra'anana, Israel

D. Zakay

Interdisciplinary Center (IDC) Herzliya, Herzliya, Israel the ways in which these two thinking modes interact. Some authors (e.g., Kahneman \& Frederick, 2002) have argued that the two modes operate sequentially, so that intuitive answers to judgment problems are generated rapidly and automatically, and then the analytical mode may initiate a process of monitoring, endorsing, correcting, or overriding the initial intuitive response. Yet other researchers (e.g., Damasio, 1994; Epstein, 1994; Glöckner \& Betsch, 2008; Sloman, 1996) have suggested that the two thinking modes work in parallel and are used to different extents depending on the decision environment. Evans also pointed out that various definitions of intuitive processes have been used in the literature. For example, some authors (e.g., Sloman, 1996) have ignored emotion altogether, while others (e.g., Damasio, 1994; Epstein, 1994) have linked intuition to emotional thinking and have emphasized the fact that intuition relies on feelings as information, whereas analytical thinking involves reasoned cost-benefit assessment, which relies on rules rather than emotion.

To the present day, there is an ongoing debate concerning the relative advantage of each mode of thinking in decision making. Studies show contradictory results. Some studies show that the intuitive mode is inferior to the analytical mode, so that intuition and/or emotion may be a source of faulty decisions (MacGregor \& Amstrong, 1994; MacGregor, Lichtenstein, \& Slovic, 1988; Tversky \& Kahneman, 1974). Other studies demonstrate that the intuitive mode yields equal or better decisions, in comparison with the analytical mode. Hence, they counsel heeding intuition and avoiding "overthinking" (Betsch, Plessner, Schwieren, \& Gütig, 2001; Dijksterhuis, Bos, Nordgren, \& van Baaren, 2006; Lee, Amir, \& Ariely, 2009; Pham, 2004; Usher, Rusou, Weyers, Brauner, \& Zakay, 2011; Wilson et al., 1993; Zakay \& Wooler, 1984).

Identifying the circumstances under which each thinking mode is preferable might help in understanding the advantages of each mode. In this respect, several researchers have highlighted the importance of one 
moderating factor, which is the compatibility between thinking mode and task characteristics (e.g., Hammond, Hamm, Grassia, \& Pearson, 1987).

\section{The effect of decision task characteristics}

Hammond et al. (1987) were the first to suggest that performance is best when the mode of a person's thinking matches the task. These researchers proposed that the nature of the task could be more intuitive or more analytical, depending on task characteristics. The characteristics of intuitive tasks include high familiarity, pictorial presentation, subjective measure, and unavailability of an organizing principle or algorithm to integrate cues. Analytical tasks are characterized by quantitative presentation, objective measures, and an organizing principle readily available. Wilson and Schooler (1991) stated that analytical deliberation is adequate for tasks that involve objective, easily verbalized attributes but is less adequate for tasks that involve a large affective component or in which the dimensions of the stimuli are ill-defined. Epstein (1994) emphasized that the intuitive mode is more adequate when nonverbal information and/or concrete stimuli are involved, whereas the analytical mode is more adequate when abstract problems are solved through symbols (numbers and words) and logical inference. According to him, the intuitive mode is used when processing emotion-arousing stimuli that represent events in a manner similar to how they are experienced in real life. Hogarth (2005) proposed that tasks are more likely to be processed through the intuitive mode when their context and form promote visual reasoning, whereas Dijksterhuis and Nordgren (2006) stated that tasks in which participants must follow strict rules are performed best through analytical deliberation.

Despite these extensive theoretical hypotheses, relatively little experimental work has directly examined the association between thinking modes and task characteristics. Hammond et al. (1987) investigated how experienced highway engineers make various decisions. These authors assumed that judging the capacity of highways is an analytical task, determining the safety of highways is a semianalytical task, and deciding on aesthetic aspects of highways is an intuitive task. In this study, engineers made better judgments when the mode of thought was compatible with the task at hand. However, they manipulated the thinking modes in their study by using different formats of information presentation. This, however, could create a confound by conveying different levels of information. For example, mathematical formulas that are used to induce analytical thinking cannot actually deliver the same information on the aesthetic aspects of highways as the film strips used to induce intuitive thinking.
McMackin and Slovic (2000) further demonstrated the importance of the match between thinking mode and task but used different measures to assess performance in each task, precluding an analysis of the thinking-mode-task interaction.

\section{The present research}

We assume that the relative advantage of each thinking mode in decision making depends on the compatibility between thinking mode and task. To test this assumption, we pitted intuition and analytical thinking against each other within either intuitive or analytical tasks, comparing decision making in a factorial design.

\section{Thinking mode manipulation}

In order to meet the challenge posed by the diversity of viewpoints regarding the nature of dual thinking, two different thinking manipulations, each congruent with a different theoretical approach, were employed. Experiment 1 relied on Wilson and Schooler's (1991) manipulation and contrasted direct choice with a deliberative analysis of reasons. This paradigm is based on a dual-processing hypothesis according to which intuitive thinking is the default mode, automatically generating impressions that can then be discarded by conscious effortful reasoning. Experiment 2 relied on Pham, Cohen, Pracejus, and Hughes's (2001) method, contrasting choices based on feelings with choices made through nonaffective deliberation. This paradigm reflects the explicit linkage of emotion and affect to intuition (Damasio, 1994; Epstein, 1994).

\section{Task selection}

Experiment 1 contrasted the assessment of faces with the evaluation of arithmetical multiplications. Experiment 2 compared the assessment of urban scenes' photographs with the evaluation of numerical averages. Judgment of faces and urban photographs were used for the intuitive tasks; since the stimuli are pictorial and concrete, involve real-life experience, and are likely to be mediated by affective responses, the different dimensions of the stimuli are not easily verbalized, the task is measured subjectively, and an organizing principle or algorithm to integrate these dimensions is not available. In contrast, evaluations of numerical multiplications and of numerical averages were chosen for the analytical tasks; because these stimuli are abstract and symbolic, the tasks are measured objectively, involving the application of welldefined logical rules, and an organizing principle is readily available. 
Transitivity as the dependent measure

Following Lee et al. (2009), we looked at transitivity in pairwise choices. Transitivity is one of the major principles of rationality that stands at the basis of the development of decision theory (von Neumann \& Morgenstern, 1948). This principle implies that for any three alternatives (A, $\mathrm{B}, \mathrm{C})$, if $\mathrm{A}$ is judged as better than B and B is judged as better than $C$, then $A$ should also be judged as better than C. If individuals are inconsistent or prone to error in decision making, they will commit many violations of transitivity. In contrast, if there is no error in decision making (or if the error rate is very low) and if individuals evaluate the different options consistently, there will be no (or very few) violations of transitivity (Lee et al., 2009). Here, we look for more consistency when thinking mode matches task, thus expecting fewer transitivity violations in such cases. The use of a single measure for both tasks enables not only the identification of comparable simple effects, but also the assessment of the thinking mode $\times$ task interaction.

\section{Experiment 1}

The experiment consisted of four conditions: two thinking modes (intuitive and analytical), compared across subjects, and two tasks (assessing faces and evaluating arithmetical multiplications), examined within subjects. We slightly adapted Wilson and Schooler's (1991) manipulation so that participants in the intuitive condition were instructed to rely on general impressions and gut feelings when making their decisions, as opposed to no specific instructions in the original study.

\section{Method}

\section{Participants and design}

Forty-seven undergraduate students from the Interdisciplinary Center (IDC) at Herzliya, Israel were randomly assigned to one of the thinking conditions (intuitive or analytical). Each participant performed two choice tasks: one in regard to faces and the other in regard to arithmetical multiplications.

\section{Materials}

Assessment of faces was examined with nine color photographs of male faces. Evaluation of arithmetical multiplication was examined with nine multiplications in which a single-digit number was multiplied by a twodigit number. For each task, 36 stimuli pairs were constructed by creating all possible pairwise combinations of the nine stimuli (see Fig. 1). Within each pair, the stimuli were centered horizontally in the middle of the screen, and a choice criterion was presented above them in the upper middle part of the screen. The choice criterion for the assessment of faces was "Which of the face seems nicer to you?" The choice criterion for the evaluation of arithmetical multiplication was "Which of the multiplications seems larger to you?" The term "seems" was selected because it does not direct participants to use a specific thinking mode.

\section{Procedure}

The order in which participants performed the two decision tasks was counterbalanced. Before each task, participants were told that they would be presented with pairs of photographed faces (or arithmetical multiplications) and that, within each pair, they would be asked to choose the face that seemed nicer to them (or the multiplication that seemed larger to them). Participants in the analytical conditions were also told, "Before making your choice you will be asked to specify the reasons that made you evaluate each face as nice/not nice (or each multiplication as large/small)."

Pairs of stimuli (faces or multiplications) were presented to participants in a random order. In the analytical condition, after each pair had been presented, and before participants made their choice, they were asked to type into a text box reasons for evaluating how nice each face was (or how large each

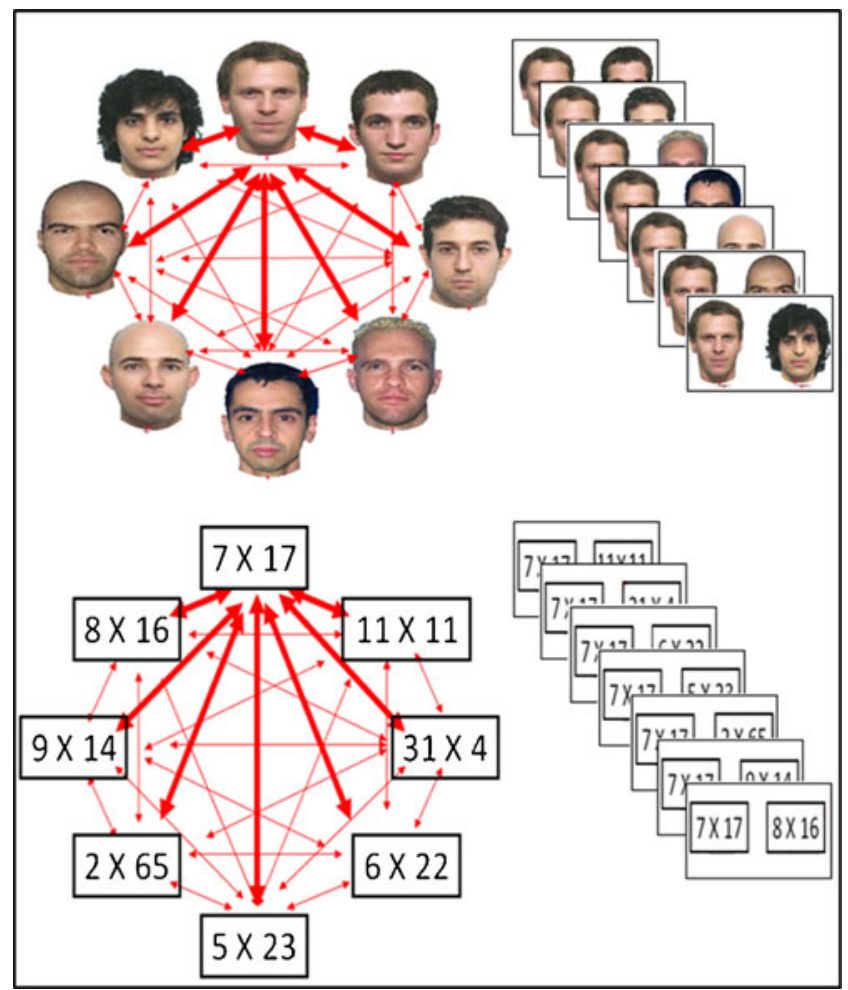

Fig. 1 Illustration of stimuli pairing in Experiment 1 
multiplication problem was). Choice was made by pressing either 1 or 2 (see Fig. 2). In the intuitive condition, participants were simply asked to make their choice. After each choice was made, the screen was cleared, and the next trial began

Results and discussion

Following Lee et al. (2009), we computed the number of transitivity violations committed by participants, in the form of three-way preference cycles (e.g., for each subgroup of three stimuli $x, y$, and $z$, if $x \geq y, y \geq z$, and $z \geq x$, a transitivity violation was made). Figure 3 presents the mean number of transitivity violations in each of the four conditions.

A two (thinking mode) $\times$ two (task type) repeated analysis of variance (ANOVA) was conducted to examine differences in the average number of transitivity violations across conditions. This analysis revealed a significant main effect of thinking mode, $F(1,45)=11.17, p<.005, \eta^{2}=.2$, and a significant main effect of task type, $F(1,45)=49.08$, $p<.000001, \eta^{2}=.52$, as well as a significant interaction between thinking mode and task type, $F(1,45)=16.8$, $p<.0005, \eta^{2}=.27$. To investigate our hypothesis that the relative advantage of the intuitive and analytical modes was contingent on task characteristics, we further conducted several planned comparisons. Within the facial task, fewer transitivity violations were made in the intuitive condition $(M=2.33, S D=2.26)$ than in the analytical condition $(M=$ $4.09, S D=3.4)$, and the effect was statistically significant, $F(1,45)=4.38, p<.05$. In contrast, on the multiplication task, fewer transitivity violations were made in the analytical condition $(M=7.17, S D=6.11)$ than in the intuitive condition $(M=14.12, S D=5.27)$. This difference was also statistically significant, $F(1,45)=17.49, p<.0005$.

\section{Experiment 2}

Experiment 2 was designed to replicate and generalize the findings of Experiment 1, with two major modifications in both choice tasks and procedure. With regard to choice tasks, we used assessment of scenes' photographs rather than assessment of faces and evaluation of averages rather than evaluation of multiplication problems. With regard to the procedure, we made use of Pham et al.'s (2001) manipulation in which the required assessment is more explicit.

Method

\section{Participants and design}

Twenty undergraduates from the IDC at Herzliya, Israel were randomly assigned to either the intuitive or the analytical thinking condition. Each participant performed the two choice tasks, in a random order.

\section{Materials}

Assessment of scenes' photographs was examined with eight black-and-white photographs of urban scenes. Evaluation of averages was examined with eight groups of 6 two-digit numbers. For each task, 28 stimuli pairs were constructed by creating all possible pairwise combinations of the eight stimuli. Within each pair, the stimuli were centered horizontally in the middle of the screen, and a choice criterion was presented above them in the upper middle part of the screen. The choice criterion for the assessment of scenes was either "Which picture feels better?" (intuitive condition) or "Which picture is better?" (analytical condition). The choice criterion for the evaluation of averages was either "The average of which group feels larger?" (intuitive condition) or "The average of which group is larger?" (analytical condition). This variation of terms (feel/is) was selected to further enhance the intuitive/analytic manipulation. In addition, below the stimuli, participants saw the phrase "Please rely on your overall impressions and your feelings. Avoid analytical assessments" (intuitive condition) or the phrase "Please base your evaluations on analytical assessments. Ignore your feelings and first impressions" (analytical condition) (Fig. 4).

\section{Procedure}

Before each task, participants were told that they would be presented with pairs of photographs (or sets of numbers). Following Pham et al.'s (2001) method, participants
Fig. 2 Examples of the two tasks in Experiment 1
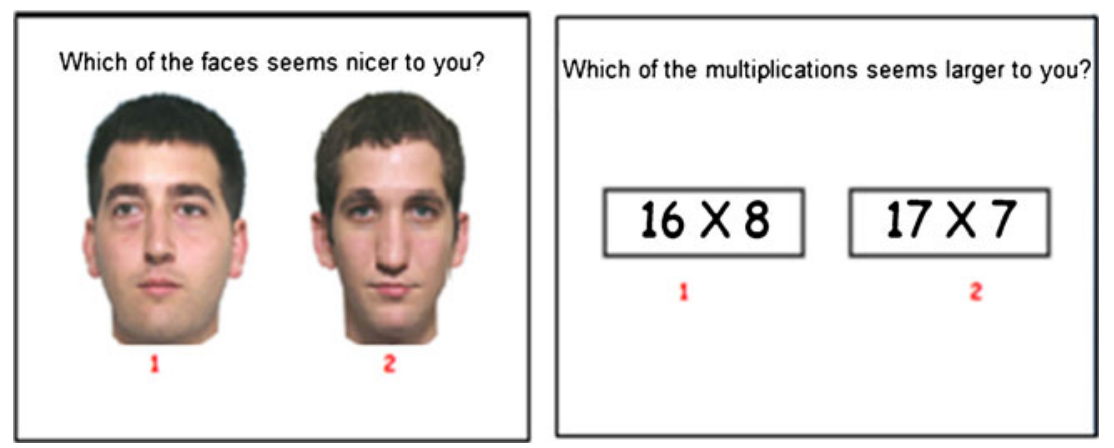


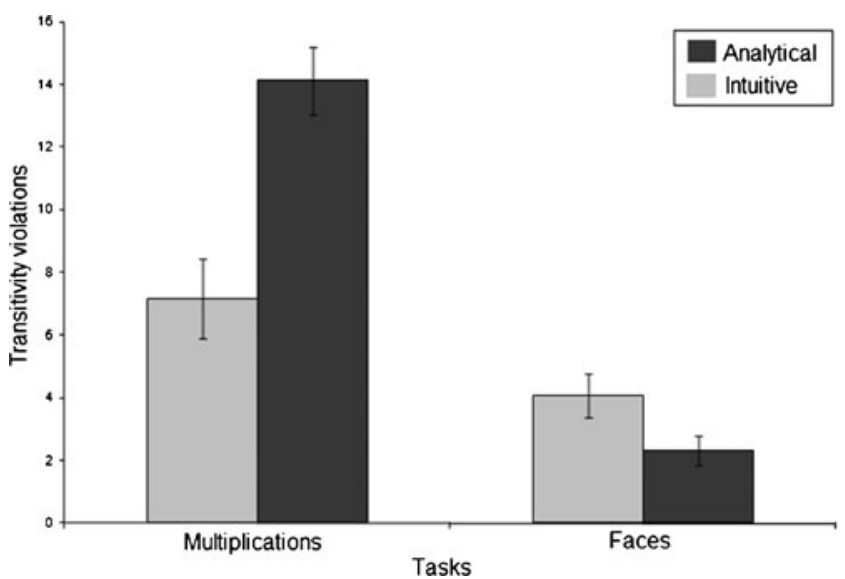

Fig. 3 The mean number of transitivity violations in Experiment 1 . The $y$-axis represents the number of transitivity violations. The more violations made, the less consistent are the decisions.

in the intuitive condition were explicitly asked to concentrate on their feelings in reaction to the stimuli, avoid analytical deliberation, and choose the photograph that felt better (or the group of numbers whose average felt higher). Participants in the analytical condition were asked to ignore their feelings in reaction to the stimuli, base their judgments on an analytical assessment of each alternative, and choose the best photograph or the group of numbers whose average was higher. Following the initial instructions, the pairs of stimuli (scenes or numbers) were presented to participants in a random order.
Results and discussion

Figure 5 presents the mean number of transitivity violations in each of the four conditions.

A two (thinking mode) $\times$ two (task type) repeated ANOVA was conducted to examine differences in the number of transitivity violations across conditions. This analysis yielded a significant main effect of task type, $F(1,18)=27.43, p<.0001, \eta^{2}=.604$, as well as a significant interaction between thinking mode and task, $F(1,18)=17.33, p<.001, \eta^{2}=.49$. To investigate our hypothesis that the relative advantage of the intuitive and analytical modes was contingent on task characteristics, we further conducted several planned comparisons. On the photographs, there were fewer transitivity violations when participants made intuitive judgments $(M=1.0, S D=1.49)$ than when they made analytical judgments $(M=2.9, S D=2.18)$, and the difference between these means was statistically significant, $F(1$, $18)=5.16, p<.05$. In contrast, when judging averages, participants made fewer transitivity violations in the analytical condition $(M=3.7, S D=2.83)$ than in the intuitive condition $(M=8.0, S D=4.19)$, and this difference was statistically significant, $F(1,18)=7.23$, $p<.05$.

Next, we analyzed the reaction times (RTs) of responses in all four conditions through a two (thinking modes) $\times$ two (task) repeated ANOVA. This analysis revealed a significant main effect of thinking mode, $F(1,18)=21.21, p<.0005$, and
Fig. 4 Examples of the stimulus materials in Experiment 2

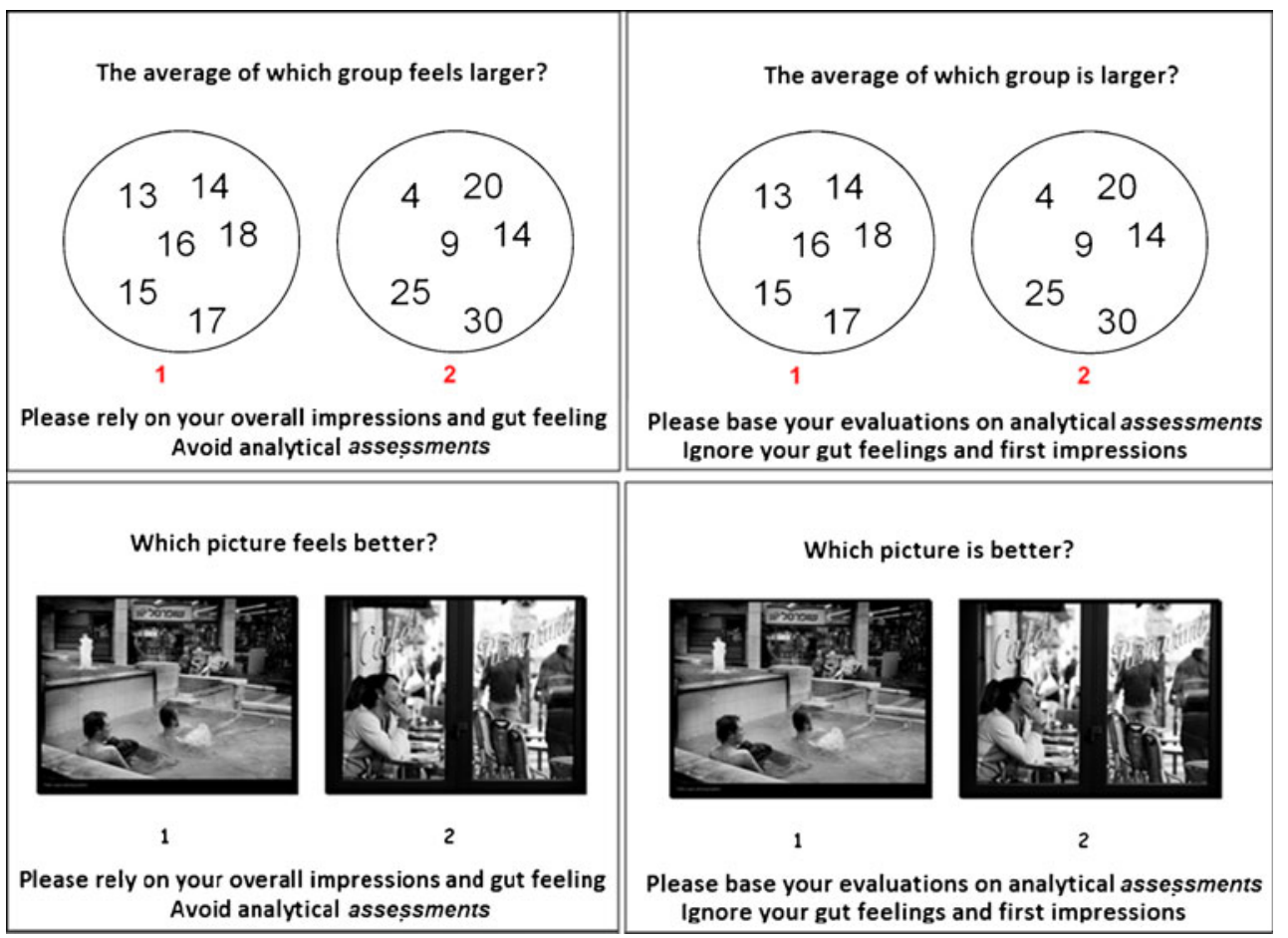




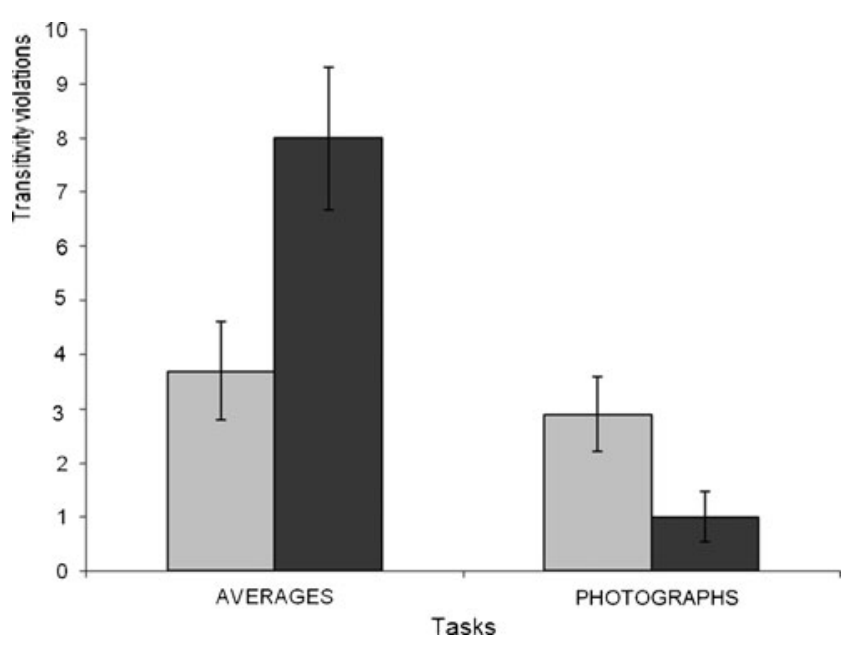

Fig. 5 The mean number of transitivity violations in Experiment 2

a significant main effect of task type, $F(1,18)=6.81, p<.05$, with a marginal interaction between thinking mode and task type, $F(1,18)=4.31, p=.052$. RT on both tasks was shorter for intuitive judgments than it was for analytical judgments. Thus, RT for deciding which scene felt better $(M=3,141 \mathrm{~ms}$, $S D=1,411)$ was shorter than was RT for deciding which scene was better ( $M=6,584 \mathrm{~ms}, S D=3,289$; Fisher LSD, $p=.18)$. Similarly, RT for deciding which average felt larger was shorter $(M=4,171 \mathrm{~ms}, S D=1,590)$ than was RT for deciding which average was larger $(M=15,649 \mathrm{~ms}, S D=10,574$; Fisher LSD, $p<.005$ ).

The results of the RT analysis are consistent with dualprocess theories that view intuition as fast and deliberation as time consuming (e.g., Kahneman, 2003). The significant difference between RTs for photographs and averages may stem from the intuitive nature of visual processing (Hogarth, 2005), as opposed to the deliberative nature of mathematical operations.

Note, though, that participants may have understood the terms feels/is differentially within each task (photographs vs. averages). To examine this possibility, we conducted an additional study $(N=20)$ in which the two thinking manipulations made use of the same neutral choice criteria ("Which picture seems better to you" and "The average of which group seems higher to you"). Overall, the results obtained in this study closely paralleled those of the original manipulation. A two (thinking mode) $\times$ two (task type) repeated ANOVA revealed a significant main effect of task type, $F(1,18)=25.82, p<.0001$, $\eta^{2}=.59$, as well as a significant interaction between thinking mode and task type, $F(1,18)=13.29, p<.005, \eta^{2}=.43$. We further conducted several planned comparisons. On the photograph task, fewer transitivity violations were made on intuitive judgments $(M=1.1, S D=1.66)$, so that they were more consistent than were analytical judgments $(M=2.7$, $S D=2.21$ ), and this difference was statistically significant, $F(1,18)=3.34, p<.05$, one-tailed. On the average task, more transitivity violations were made on intuitive judgments $(M=9.0, S D=4.64)$ than on analytical judgments $(M=4.0, S D=3.4)$, and this difference was statistically significant, $F(1,18)=7.55, p<.05$. The relative advantage of the intuitive and analytical modes was contingent on task characteristics. This pattern of results replicates our main finding of an interaction between thinking mode and task characteristics.

\section{General discussion}

In view of the controversy about the relative advantage of the two thinking modes in decision making, (Gilovich \& Griffin, 2010; Hogarth, 2005; Inbar, Cone, \& Gilovich, 2010), the question posed by the present research is whether the relative capability of the analytical and intuitive modes to make good decisions is mediated by the nature of the decision task (analytical or intuitive). Together, the experiments conducted here provide strong support for the hypothesis that the compatibility between thinking mode and task characteristics determines which mode is optimal in each case. On pictorial tasks, intuitive thinking resulted in fewer transitivity violations (more consistency across choices) than did analytical thinking, whereas on numerical tasks, analytical deliberation led to more consistency than did intuitive thinking. The validity of these findings is strengthened by the parallel patterns of outcomes obtained across three different studies reported here, under different sets of intuitive and analytical tasks, as well as by different manipulations.

These results strengthen and extend the findings reported by Hammond et al. (1987) and by McMackin and Slovic (2000). Since previous results were subject to alternative explanations, the present study was designed to avoid similar pitfalls. (1) Information was presented in a uniform format across conditions, unlike in Hammond et al. (1987). (2) A uniform measure was used for both the intuitive and the analytical tasks. As has been pointed out by Payne, Bettman, and Johnson (1993), only a uniform measure can provide a true comparison that involves no alternative explanations of the use of each thinking mode. (3) Looking at transitivity relations and violations across choices offers a measure of consistency that was missing in previous studies whose focus was on simple effects alone (e.g., McMackin \& Slovic, 2000).

The present results can resolve some of the contradictory findings found in the literature. Note that many studies that stressed the strength of intuition drew on stimuli that presented events in a manner similar to real life, mostly using pictorial stimuli (e.g., Levine, Halberstadt, \& Goldstone, 1996; Wilson et al., 1993). Thus, stimuli that showed the superiority of intuitive thinking were likely to involve a 
larger affective component, offering no algorithm by which to integrate cues (e.g., Dijksterhuis, 2004; Dijksterhuis et al., 2006; Wilson et al., 1993; Wilson \& Schooler, 1991). In contrast, studies in which analytical thinking was found to be superior (e.g., MacGregor et al., 1988; Tversky \& Kahneman, 1974) used abstract deliberative tasks that involved numerical stimuli and presented an objective criterion as well as logical rules.

To conclude, we propose that the debate as to whether decision quality is best when using intuitive or analytical thinking might be clarified once each thinking mode is examined within a task that favors such thinking.

\section{References}

Betsch, T., Plessner, H., Schwieren, C., \& Gütig, R. (2001). I like it but I don't know why: A value-account approach to implicit attitude formation. Personality and Social Psychology Bulletin, 27, 242253.

Damasio, A. R. (1994). Descartes' error: Emotion, reason, and the human brain. New York: Avon.

Dijksterhuis, A. P. (2004). Think different: The merits of unconscious thought in preference development and decision making. Journal of Personality and Social Psychology, 87, 586-598.

Dijksterhuis, A. P., Bos, M. W., Nordgren, L. F., \& van Baaren, R. B. (2006). On making the right choice: The deliberation without attention effect. Science, 311, 1005-1007.

Dijksterhuis, A. P., \& Nordgren, L. F. (2006). A theory of unconscious thought. Perspectives on Psychological Science, 1, 95-109.

Epstein, S. (1994). Integration of the cognitive and psychodynamic unconscious. American Psychologist, 49, 709-724.

Evans, J. S. B. T. (2008). Dual-processing accounts of reasoning, judgment, and social cognition. Annual Review of Psychology, $59,255-278$.

Gilovich, T., \& Griffin, D. W. (2010). Judgment and decision making. In D. T. Gilbert \& S. T. Fiske (Eds.), The handbook of social psychology (5th ed., pp. 542-588). New York: McGraw-Hill.

Glöckner, A., \& Betsch, T. (2008). Modeling option and strategy choices with connectionist networks: Towards an integrative model of automatic and deliberate decision making. Judgment and Decision Making, 3, 215-228.

Hammond, K. R., Hamm, R. M., Grassia, J., \& Pearson, T. (1987). Direct comparison of the relative efficiency on intuitive and analytical cognition. IEEE Transactions on Systems, Man, and Cybernetics, 17, 753-770.

Hogarth, R. M. (2005). Deciding analytically or trusting your intuition? The advantages and disadvantages of analytic and intuitive thought. In T. Betsch \& S. Haberstroh (Eds.), The routines of decision making (pp. 67-82). Mahwah: Erlbaum.

Inbar, Y., Cone, J., \& Gilovich, T. (2010). People's intuitions about intuitive insight and intuitive choice. Journal of Personality and Social Psychology, 99, 232-247.

Kahneman, D. (2003). A perspective on judgment and choice: Mapping bounded rationality. American Psychologist, 58, 697720.

Kahneman, D., \& Frederick, S. (2002). Representativeness revisited: Attribute substitution in intuitive judgment. In T. Gilovich, D. Griffin, \& D. Kahneman (Eds.), Heuristics and biases (pp. 4981). New York: Cambridge University Press.

Lee, L., Amir, O., \& Ariely, D. (2009). In search of homo economicus: Cognitive noise and the role of emotion in preference consistency. Journal of Consumer Research, 36, 173-187.

Levine, G. M., Halberstadt, J. B., \& Goldstone, R. L. (1996). Reasoning and the weighing of attributes in attitude judgments. Journal of Personality and Social Psychology, 70, 230-240.

MacGregor, D. G., \& Armstrong, J. S. (1994). Judgmental decomposition: When does it work? International Journal of Forecasting, 10, 495-506.

MacGregor, D. G., Lichtenstein, S., \& Slovic, P. (1988). Structuring knowledge retrieval: An analysis of decomposed quantitative judgments. Organizational Behavior and Human Decision Processes, 42, 303-323.

McMackin, J., \& Slovic, P. (2000). When does explicit justification impair decision making? Journal of Applied Cognitive Psychology, 14, 527-541.

Payne, J. W., Bettman, J. R., \& Johnson, E. J. (1993). The adaptive decision maker. Cambridge: Cambridge University Press.

Pham, M. T. (2004). The logic of feeling. Journal of Consumer Psychology, 14, 360-369.

Pham, M. T., Cohen, J. B., Pracejus, J. W., \& Hughes, G. D. (2001). Affect monitoring and the primacy of feelings in judgment. Journal of Consumer Research, 28, 167-188.

Sloman, S. A. (1996). The empirical case for two systems of reasoning. Psychological Bulletin, 119, 3-21.

Tversky, A., \& Kahneman, D. (1974). Judgment under uncertainty: Heuristics and biases. Science, 185, 1124-1131.

Usher, M., Russo, Z., Weyers, M., Brauner, R., \& Zakay, D. (2011). The impact of the mode of thought in complex decisions: Intuitive decisions are better. Frontiers in Psychology, 2, 1-13.

von Neumann, J., \& Morgenstern, O. (1947). Theory of games and economic behavior. Princeton: Princeton University Press.

Wilson, T. D., Lisle, D., Schooler, J. W., Hodges, S. D., Klaaren, K. J., \& LaFleur, S. J. (1993). Introspecting about reasons can reduce post-choice satisfaction. Personality and Social Psychology Bulletin, 19, 331-339.

Wilson, T. D., \& Schooler, J. W. (1991). Thinking too much: Introspection can reduce the quality of preferences and decisions. Journal of Personality and Social Psychology, 60, 181-192.

Zakay, D., \& Wooler, S. (1984). Time pressure, training, and decision effectiveness. Ergonomics, 27, 273-284. 